\title{
Do activity monitors increase physical activity in adults with overweight or obesity? A systematic review and meta-analysis
}

\author{
Herman J. de Vries ${ }^{1,2}$, Thea J.M. Kooiman³, Miriam W. van Ittersum4, Marco van Brussel', Martijn de Groot ${ }^{3,5}$.
}

1 Clinical Health Sciences, Utrecht University / University Medical Center Utrecht, the Netherlands;

2 Paramedics PT center, Assen, the Netherlands;

3 Research Group Healthy Ageing, Allied Health Care and Nursing, Hanze University of Applied Sciences, Groningen, the Netherlands;

4 School of Health Care Studies, Physiotherapy Department, Hanze University of Applied Sciences, Groningen, the Netherlands;

5 Quantified Self Institute, Hanze University of Applied Sciences, Groningen, the Netherlands.

\section{Background}

Activity monitors might be useful tools in interventions for people with obesity. The objective of this study was to systematically assess contemporary knowledge regarding behavioral physical activity interventions including an activity monitor (BPAI+) in adults with overweight or obesity.

\section{Methods}

PubMed/MEDLINE, Embase, CINAHL, PsycINFO, CENTRAL and PEDro were searched for eligible full text articles up to July 1st 2015. Methodological quality was assessed independently using the Cochrane Collaboration's tool for risk of bias.
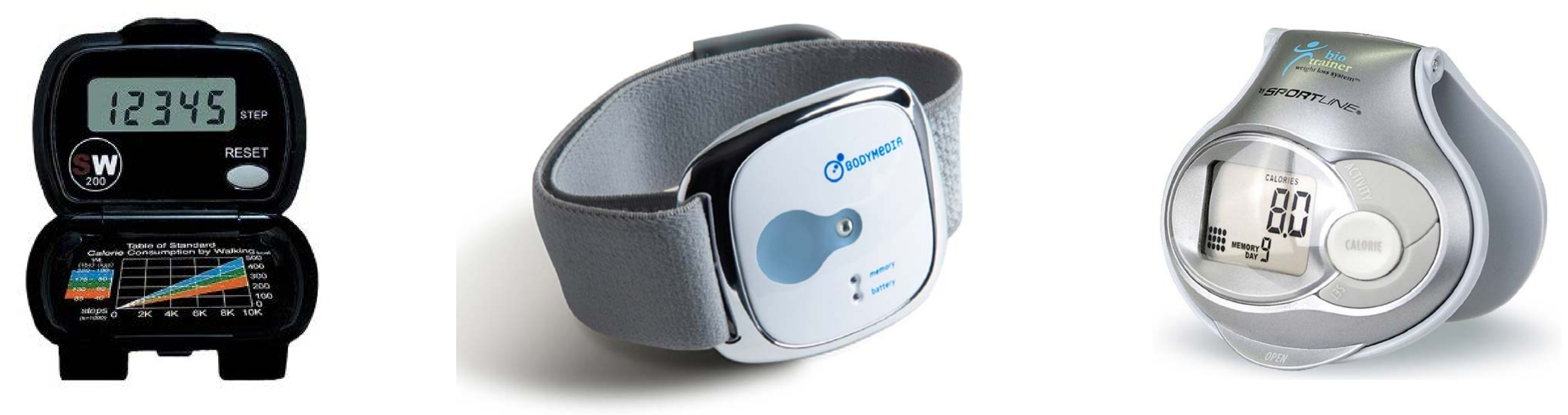

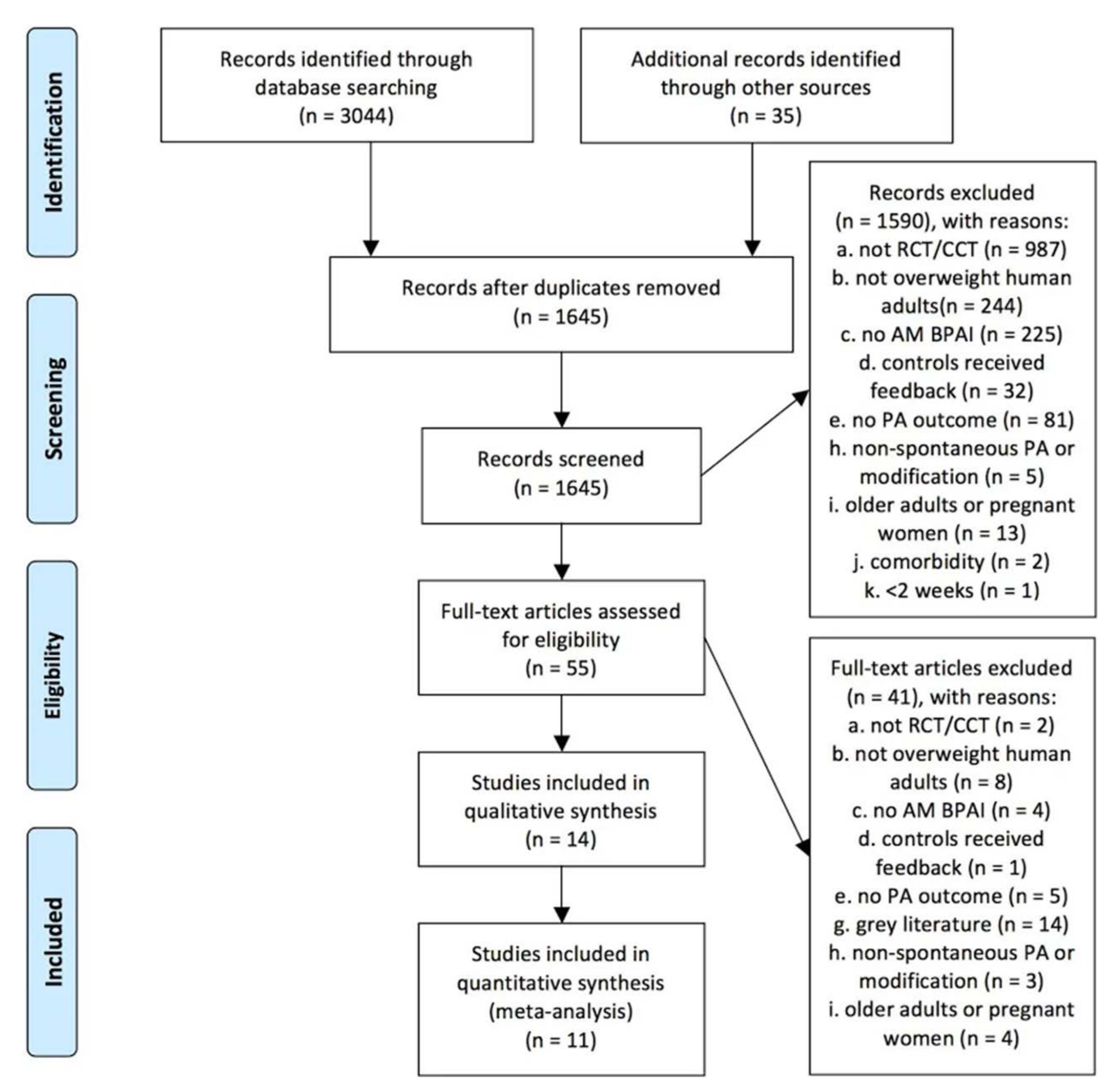

Figure 1: Flowchart of selected studies.

\section{Results}

Fourteen studies (1157 participants) were included for systematic review and eleven for meta-analysis. A positive trend in BPAIt effects on several measures of physical activity was ascertained compared to both waitlist or usual care (WL/UC) and behavioral physical activity interventions without an activity monitor (BPAI-). No convincing evidence of the effects of activity monitor use on weight loss was found when comparing BPAI+ to BPAI-.

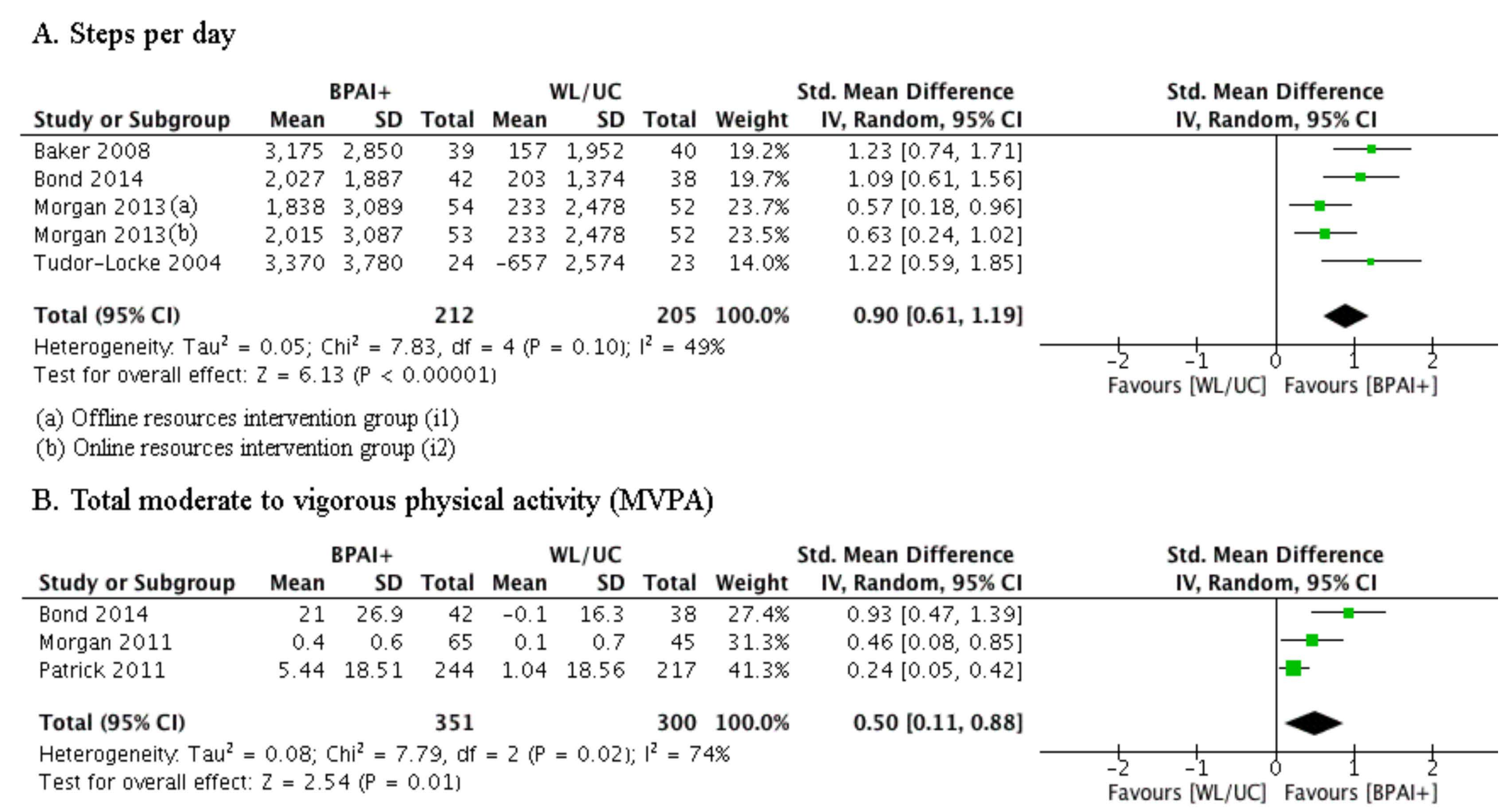

Figure 2: Meta-analysis for BPAI+ vs. WL/UC.

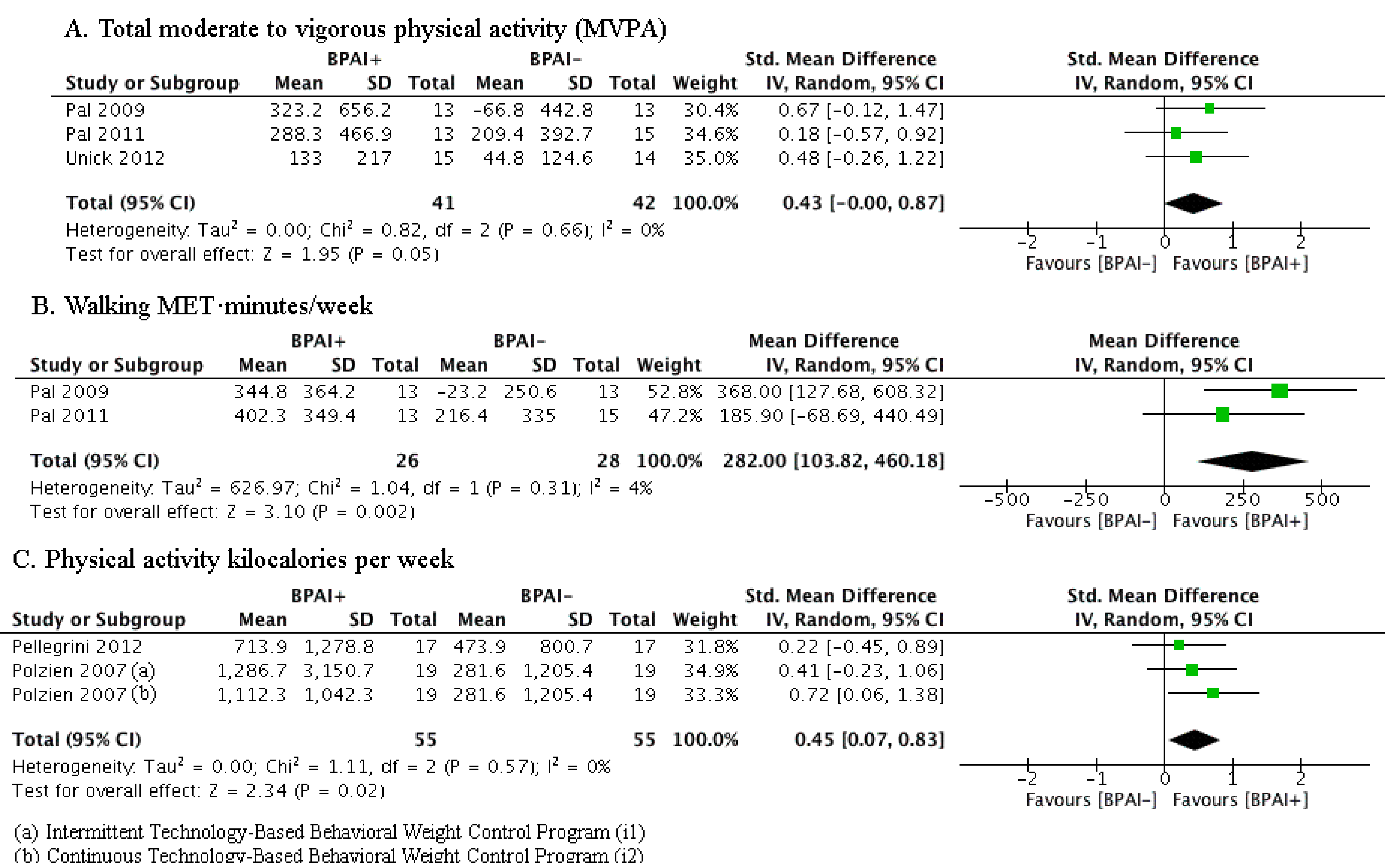

Figure 3: Meta-analysis for BPAI+ vs. BPAI-.

\section{Conclusion}

Behavioral physical activity interventions with an activity monitor increase physical activity in adults with overweight or obesity. Also, adding an activity monitor to behavioral physical activity interventions appears to increase the effect on physical activity, although current evidence has not yet provided conclusive evidence for its effectiveness.

\section{Contact:}

Herman de Vries: hermandevries@gmail.com Thea Kooiman: t.j.m.kooiman@pl.hanze.nl 